Article

\title{
Preparation, Stability and Local Piezoelectrical Properties of P(VDF-TrFE)/Graphene Oxide Composite Fibers
}

\author{
Maxim Silibin ${ }^{1,2,3, *}$, Dmitry Karpinsky ${ }^{1,3}$, Vladimir Bystrov ${ }^{4}{ }^{\circ}$, Dzmitry Zhaludkevich ${ }^{3}$, \\ Marina Bazarova ${ }^{5}$, P. Mirzadeh Vaghefi $\left.{ }^{6}{ }^{(}\right)$, P. A. A. P. Marques ${ }^{6}\left(\mathbb{C}\right.$, Budhendra Singh ${ }^{6}$ and \\ Igor Bdikin ${ }^{6}(\mathbb{D}$ \\ 1 Institute of Perspective Materials and Technologies, National Research University of Electronic Technology, \\ 119991 Moscow, Russia \\ 2 Institute for Bionic Technologies and Engineering, I. M. Sechenov First Moscow State Medical University, \\ 119991 Moscow, Russia \\ 3 Scientific-Practical Materials Research Centre of NAS of Belarus, 220072 Minsk, Belarus \\ 4 Institute of Mathematical Problems of Biology, Keldysh Institute of Applied Mathematics RAS, \\ 142290 Pushchino, Russia \\ 5 Research and Manufacturing Complex Technology Center MIET, 119991 Moscow, Russia \\ 6 Centre for Mechanical Technology Automation, Mechanical Engineering Department and Aveiro Institute of \\ Nanotechnology (AIN), University of Aveiro, 3810-193 Aveiro, Portugal \\ * Correspondence: karpinsky@physics.by; Tel.: +375-17-284-11-68
}

Received: 2 July 2019; Accepted: 10 August 2019; Published: 13 August 2019

check for updates

\begin{abstract}
The unprecedented attributes such as biocompatibility and flexibility of macromolecular piezoelectric polymer has triggered an immense interested in scientific society for their potential exploitation in implantable electronic devices. In the present article, a theoretical and experimental investigation is done to explore the polarization behavior of composite fibers based on copolymer poly-trifluoroethylene $\mathrm{P}(\mathrm{VDF}-\mathrm{TrFE})$ and graphene oxide $(\mathrm{GO})$ with varying composition of the components is explored for its possible application in bioelectronic devices. Electromechanical properties of the PVDF/GO nanofibers were investigated using piezoresponse force microscopy (PFM) method. The switching behavior, charge states, and piezoelectric response of the fibers were found to depend on the concentration of GO up to $20 \%$. Theoretical models of PVDF chains, interacting with Graphene/GO layers has been used to explore the evolution of piezoresponse in the composite fibers. In order to compute piezoelectric coefficients, the behavior of composite in electrical fields has been modeled using software HyperChem. The experimental results are qualitatively correlated with a computed theoretical model.
\end{abstract}

Keywords: ferroelectric polymer; graphene oxide; composites; piezoelectricity; piezoresponse force microscopy

\section{Introduction}

In recent years, polymer nanocomposites fiber microstructures have received great attention due to improved physical properties, such as higher mechanical stability, improved thermal properties, non-toxicity, and more possibilities of modification than the original polymer matrix. This allows the use of these composites in various commercial applications, such as biomedical materials [1], gas filtration membranes [2], water harvesting [3-5], catalytic membrane [6], polymer electrolytes [7-9], and energy harvesting [10-13]. 
One of the best polymers such as polyvinylidene fluoride (PVDF) and copolymers of poly(vinylidene fluoride) and poly trifluoroethylene viz. P(VDF-TrFE) are perspective materials to produce flexible lightweight piezoelectric materials to be utilized in energy harvesting applications [13,14]. Organic materials which possess notable piezoresponse are highly demanded by industry to produce piezoelectric sensors, actuators, transducers as well as bioMEMS [15]. During the last years, the nanocomposites based on PVDF and graphene (graphene oxides) became a subject of particular scientific interest [16].

In spite of a number of studies dedicated to chemical, electric and pyroelectric properties of $\mathrm{P}(\mathrm{VDF}-\mathrm{TrFE})$ based nanocomposites, the effect of GO adding on the mentioned properties is still unclear. Influence of the surface and boundary effects, especially in composite materials can significantly enhance the ability to tune their fundamental properties. Adding of graphene oxide into the PVDF nanofibers leads to a change in transport properties of the composites as GO flakes act as charge trapping centers which is perspective for production of charge storage materials [17].

Many investigations focus on relevant experimental studies and theoretical modeling of the state of polarization and electrical characteristics of nanocomposites with $\mathrm{P}(\mathrm{VDF}-\mathrm{TrFE})$ under the influence of various external factors such as electric field, illumination, thermal and mechanical gradients, and will allow to reveal the mechanism of dipole ordering of the polymer matrix, changing its degree of crystallinity in the presence of graphene inclusions, and will evaluate the stability of their functional properties, especially piezoelectric and pyroelectric [18].

The main objective of the study is focused on the synthesis of nanocomposite fiber materials based on polymer polyvinylidene fluoride and its copolymers with graphene oxide using atomic/piezo force microscopy.

\section{Experimental and Computational Details}

Graphene oxide (GO) was prepared by the chemical exfoliation of graphite (Graphite powder, size $<45 \mu \mathrm{m}$, purity $\geq 99.99 \%$, Sigma-Aldrich, St. Louis, MO, USA) [19]. Briefly, a controlled reaction of graphite flakes with concentrated $\mathrm{H}_{2} \mathrm{SO}_{4}$ and $\mathrm{KMnO}_{4}$ was done to obtain individual sheets of graphene oxide. After extensive washing with distilled water, the resultant suspension was subjected to filtration and centrifugation before dialyses to remove ionic contaminants. Finally, the resulting GO slurry was dried by lyophilization to avoid aggregation.

For the composite sample preparation, copolymer of poly (vinylidene fluoride-trifluoroethylene) with $30 \%$ TrFE content was used as it favors a crystallization of PVDF directly into the ferroelectric phase. In $100 \mathrm{~mL}$ solvent of dimethyl sulfoxide (DMSO) and acetone in 80/20 ratio, 4 grams of copolymer powder was dissolved and stirred for $2 \mathrm{~h}$ at a temperature of $100^{\circ} \mathrm{C}$. The solution was then carefully filtered to remove impurities. Different mixture with varying concentrations of GO were prepared by adding dried GO powder in the prepared solvent. The mixtures were then sequentially subjected to magnetic stirring for $30 \mathrm{~min}$ at $50{ }^{\circ} \mathrm{C}$, ultrasonication for $1 \mathrm{~h}$ and magnetic stirring for $3 \mathrm{~h}$ at $50^{\circ} \mathrm{C}$ to obtain a homogeneous distribution of GO in the solution. Nanocomposite fibers were prepared by electrospinning method (NANON-01A) using P(VDF-TrFE)/GO (0-20 wt \%) solutions. The polymer composite solution placed in a plastic syringe was deposited on aluminum substrate via electrospinning method with a rate of $0.2-0.4 \mathrm{~mL} / \mathrm{h}$.

Atomic Force Microscopy (AFM) measurements were performed using Veeco Multimode Nanoscope microscope (Michigan Tech, Houghton, MI, USA). During EFM (Electric Force Microscopy, Park System, Suwon, Korea) measurements a conducting AFM tip senses an electric force gradient due to a difference in phase shift estimated at first pass (topography scan) and second pass when the tip is lifted to about $100 \mathrm{~nm}$ height. The map of the electric field gradient was constructed using the phase difference signal. To measure the work function of the sample surface the Kelvin Probe Force Microscopy (KPFM, Park System, Suwon, Korea) mode was utilized. Local piezoresponse of the fibers were measured using piezoresponse force microscopy (PFM) method [20]. 
Piezoresponse force microscopy relies on the linear strain response as a function of the external electric field. Regardless of this linear response that is inherent to materials without center of inversion symmetry, all materials dispose of a quadratic strain response in the electric field, known as electrostriction. Electrostriction, therefore, provides a local electromechanical response at the second harmonic of the $A C$ (alternating current) voltage in piezoresponse force microscopy. This electrostrictive response itself provides valuable insight into the material properties. It becomes particularly interesting for the systems where the symmetry of the crystal does not allow a distinctive mode of deflection of the AFM, especially for lateral deflections. In this scenario, this type of microscopy provides a powerful imaging tool for local material defects like misfit dislocations.

Several molecular models for PVDF (in usual $\beta$-phase, which can be in polar ferroelectric phase) and graphene oxide as common composite ferroelectrics systems were developed and investigated using software HyperChem 7.51 and 8.0 (Gainesville, FL, USA) [21]. Different computational methods were used, such as the molecular mechanics (MM) methods (including MM+, Amber, BIO CHARM), quantum mechanical (QM) self-consistent field (SCF) Hartree-Fock (HF) calculations, as well as semi-empirical methods (such as AM1, PM3, ZINDO-1), in the restricted Hartree-Fock (RHF) and unrestricted Hartree-Fock (UHF) approximations. The aim of the usage of both the MM and QM methods used for molecular modeling is to search and to obtain the minimum of total or potential energy surface (PES), of the investigated molecular systems which correspond to optimal atomic configuration of the system. The optimization of molecular systems and finding of their optimal geometry is executed in this work using the Polak-Ribere (conjugate gradient method) algorithm, which determines an optimized geometry at their minimum total energy point (using PES). These various computational methods were used for the detailed debugging, validation and testing of the models. But for final current calculations of the optimized models the next methods-BIO CHARM and PM3 in RHF approximations were used.

For randomly oriented grain the components of piezoelectric tensor $d_{\mathrm{ij}}$ are the linear combination of the tensor components associated with principal crystal axes and Euler angles [22]. For unclamped grain with electrodes, the measured piezoresponse is determined by relative piezoelectric coefficients (viz. $d_{33}, d_{31}$, and $d_{15}$ ) and also depending on the orientation of the grain at the substrate. For grains oriented along the $c$-axes the piezoelectric strain is associated with $d_{33}$ coefficient and depends on the phase shift between the applied field and obtained displacement. Zero phase shift corresponds to upward orientation of the displacement and respectively a "positive" orientation of piezoresponse signal, while 180 degree of the phase shift corresponds to "negative" orientation of the polarization in the grains.

\section{Results}

Figure 1 shows X-ray diffraction (XRD) pattern $\left(2 \theta=8-50^{\circ}\right.$ scan) for $\mathrm{P}(\mathrm{VDF}-\mathrm{TrFE})$-Graphene oxide/Al, $\mathrm{P}(\mathrm{VDF}-\mathrm{TrFE})$ and Graphene oxide. The strong peaks on these diffraction patterns corresponding to GO, $\mathrm{Al}$ and $\mathrm{P}(\mathrm{VDF}-\mathrm{TrFE})$ can distinctively be seen. The XRD patterns of GO contain broad diffraction peak (002) located at $10.3^{\circ}$. It corresponds to $d=8.15 \AA$. In the mixed sample ( $20 \% \mathrm{GO})$ we can observe the peaks from Al, P(VDF-TrFE) and GO phases. Initial P(VDF-TrFE) and $\mathrm{P}(\mathrm{VDF}-\mathrm{TrFE})$-Graphene oxide/Al fibers contain two main phases: polar active phase-named $\alpha$ and non-polar phase- $\beta$-phase [23]. 


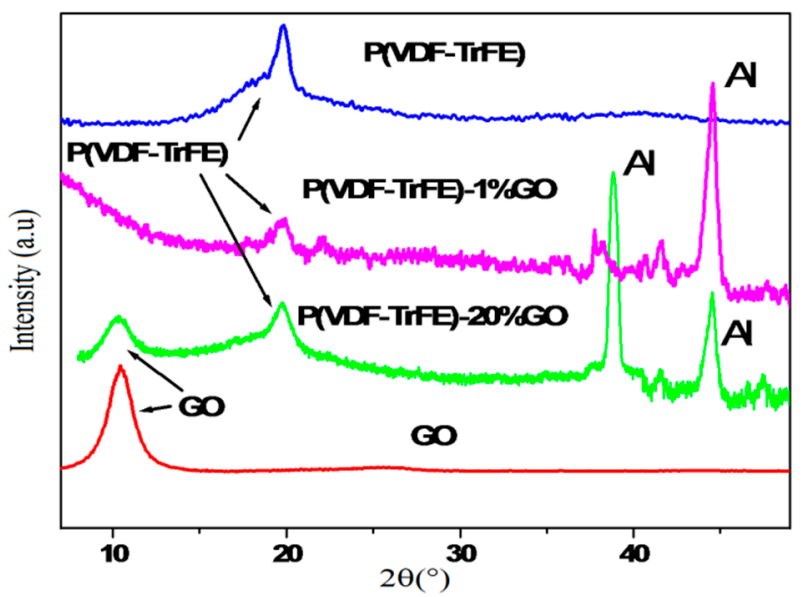

Figure 1. X-ray diffraction patterns of poly-trifluoroethylene P(VDF-TrFE), graphene oxide and $\mathrm{P}(\mathrm{VDF}-\mathrm{TrFE})$-graphene oxide/Al fibers.

The surface morphologies of the P(VDF-TrFE)-Graphene oxide/Al fibers were observed by AFM (Figures 2 and 3). The topography image of single PVDF electrospun fibers (Figure 2a) shows an average diameter of about $500 \mathrm{~nm}$.

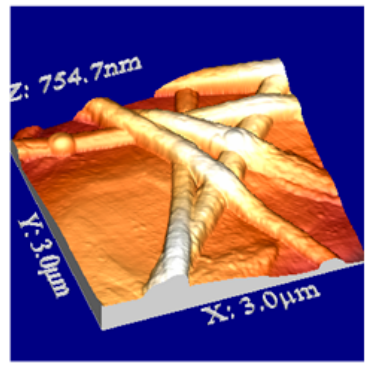

(a)

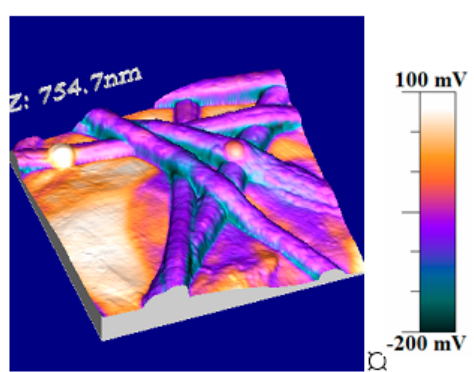

(b)

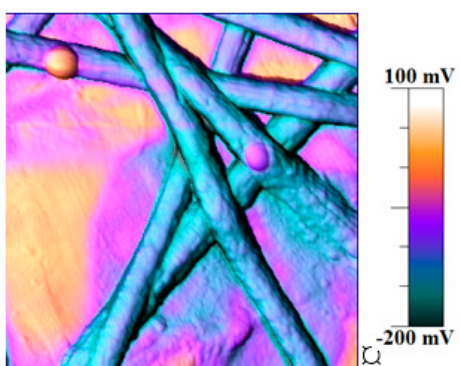

(c)

Figure 2. $\mathrm{P}(\mathrm{VDF}-\mathrm{TrFE}) /$ Graphene oxide composite fibers. (a) 3D topography image, (b,c) Topography with Kelvin Probe Force Microscopy (KPFM) image (surface potential) over-imposed (colored coded). The topography is mapped onto the third dimension (z-axis) and the recorded surface potential is color-coded; (b) 3D side view; (c) 3D top view.

The observed AFM topography image demonstrates the morphology of P(VDF-TrFE)/GO fibers. These images did not show any individual GO particles on the surface of fibers. After analyzing topographic image for each composition, average roughness (RMS roughness) of fiber surface was calculated and found to increase from 4.8 to $8.6 \mathrm{~nm}$ for an increase in GO concentration from $0 \%$ to $20 \%$, respectively. This increase in roughness could be attributed to the creation of irregular stresses during the crystallization of the fibers due to the presence of randomly distributed GO flakes having different average size.

Kelvin Probe Force Microscopy method was used to visualize the potential at the sample surface [24]. A combination of the data obtained from KPFM with AFM method was used to reveal a surface charge potential signal. Two-passes KPFM technique was performed for imaging of the contact potential difference between the AFM tip and the surface (Figure 2b,c). During the first pass of KPFM scan a topographic image is acquired [24,25]. The second pass is performed at $\sim 100 \mathrm{~nm}$ lift height tip to follow the topographic profile whereas a sum of a variable $d c$ voltage $V_{\text {tip-dc }}$ and an AC voltage, $V_{\text {tip-ac }}$ is applied to the tip. The value of $V_{\text {tip-dc }}$ voltage is adjusted to cancel the force component and thus is equal to the surface potential. To calculate the charge density value a correlation between the 
surface charge density and the obtained potential was analyzed. Figure 3 shows the topography and surface potential image as a function of GO content.

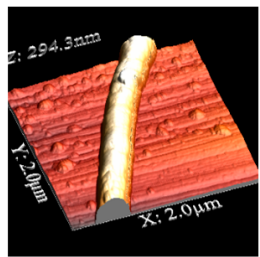

(a)

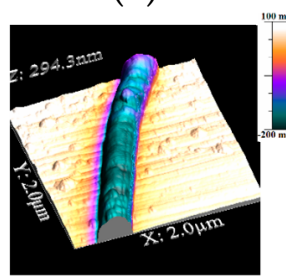

(f)

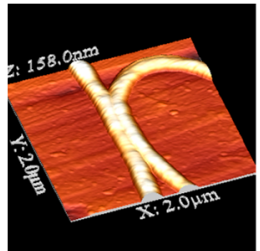

(b)

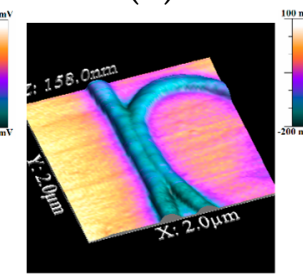

$(\mathrm{g})$

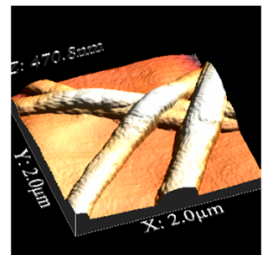

(c)

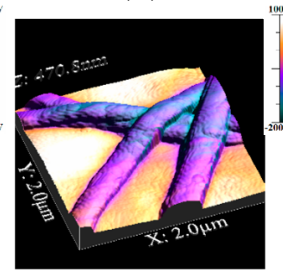

(h)

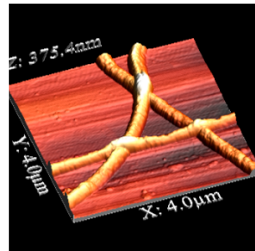

(d)

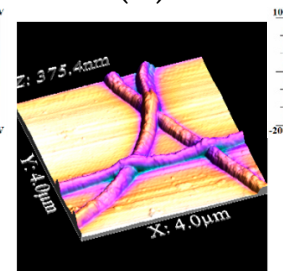

(i)

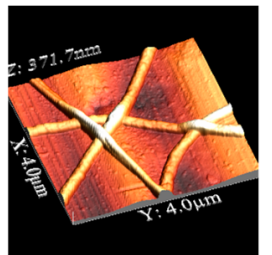

(e)

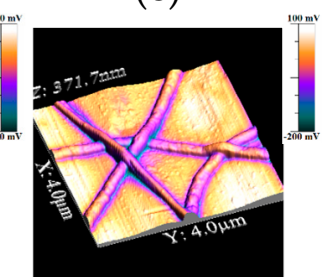

(j)

Figure 3. $\mathrm{P}(\mathrm{VDF}-\mathrm{TrFE}) /$ Graphene oxide composite fibers. (a-e) 3D topography image; (f-j) Topography with KPFM image (surface potential) over-imposed (colored coded). (a,f) $0 \%$ graphene oxide (GO); (b,g) 1\% GO; (c,h) 5\% GO; (d,i) 10\% GO; (e,j) 20\% GO.

An increase in GO content leads to an increase in the surface potential as the GO inclusions act as charge trappers which is estimated from KPFM measurements, the surface potential difference as a function of GO concentration in P(VDF-TrFE)/Graphene oxide composite fibers is presented in Figure 4.

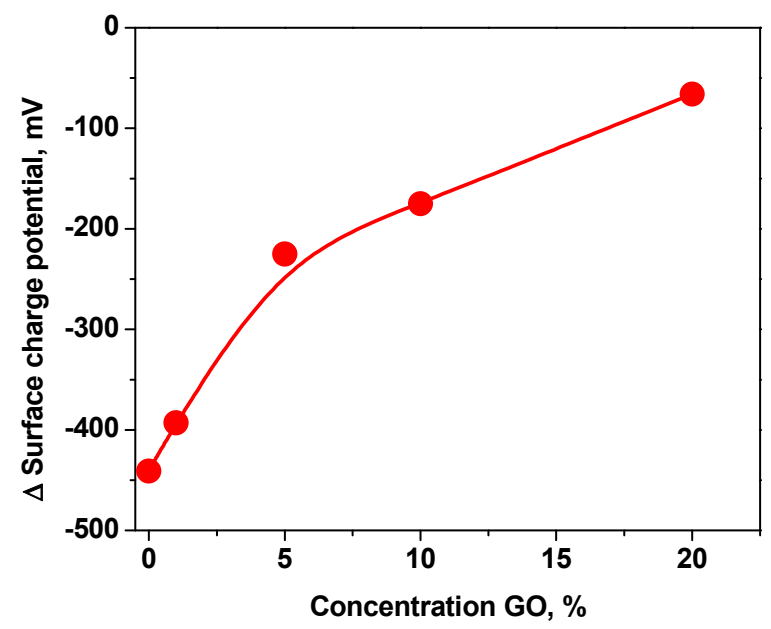

Figure 4. Variation in surface potential of $\mathrm{P}(\mathrm{VDF}-\mathrm{TrFE}) / \mathrm{Graphene}$ oxide composite fibers with GO concentration relative to aluminum foil.

In addition, depending on the distance from the center of the fiber, cross-sections perpendicular to the fibers (Figure $5 \mathrm{a}-\mathrm{c}$ ), indicates change in the surface potential. Moreover, at the edges of the fiber (position 2), the potentials are significantly reduced, which is pictorially represented in Figure $5 \mathrm{~d}$. 
(a)

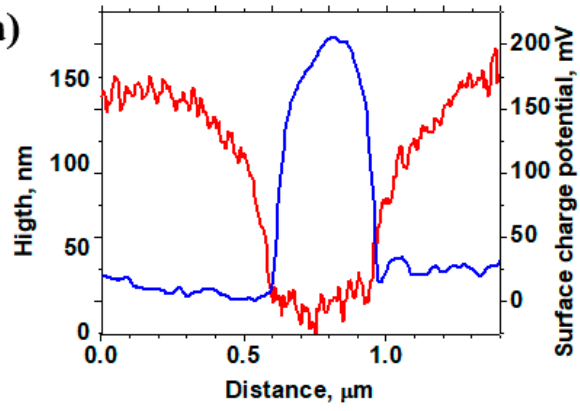

(c)

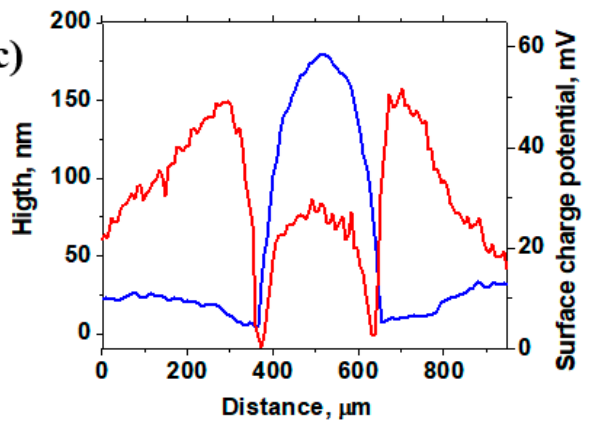

(b)
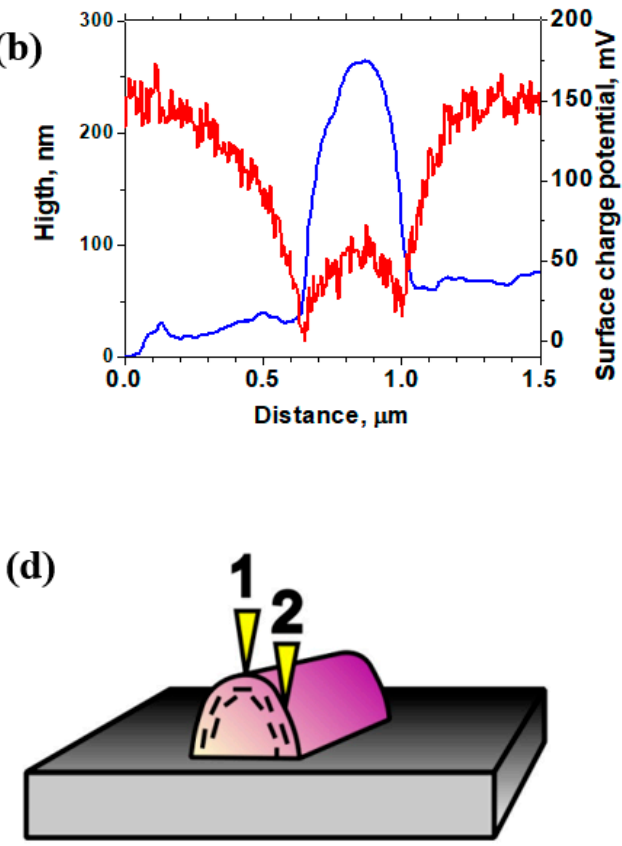

Figure 5. (a-c) Profile of the KPFM images (surface potential); (d) Schematic illustration of KPFM of $\mathrm{P}(\mathrm{VDF}-\mathrm{TrFE}) /$ Graphene oxide composite fibers. (a) $0 \% \mathrm{GO}$; (b) 5\% GO; (c) 20\% GO.

The obtained PFM loops are specific for local switching process, wherein a change in the PFM contrast is justified by the integrated piezoresponse of original domains and "unswitched" polarization. The measurements were done in the so-called pulse mode. Figure 6 shows the hysteresis loops for the fibers with various concentration of GO. Hysteresis loop acquisition was done by applying a DC voltage $\left(V_{\mathrm{dc}}\right)$ in the range of -75 to $+75 \mathrm{~V}$ with voltage sweep of 1 Volt for a short time $(1 \mathrm{~s})$ and by measuring PFM signals at each sweep voltage.

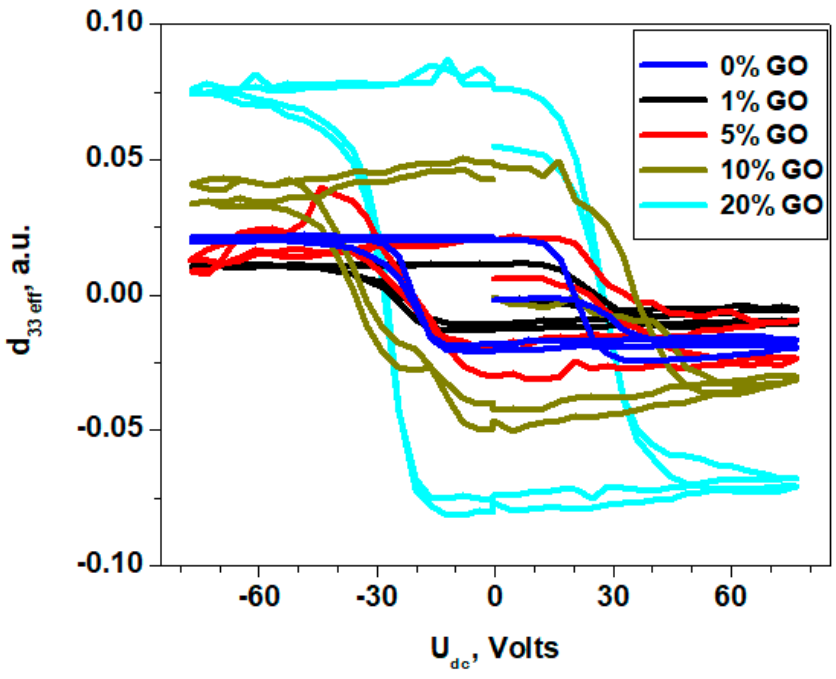

Figure 6. Piezoresponse hysteresis loops of $\mathrm{P}(\mathrm{VDF}-\mathrm{TrFE})$-graphene oxide fibers with different concentration of GO. $U_{\mathrm{ac}}=7.5 \mathrm{~V}, 40 \mathrm{kHz}$.

The loops look similar for all the fibers, which demonstrates an initial small piezoresponse with a significant offset relative to the basic level. The maximum value of the piezoelectric response signal

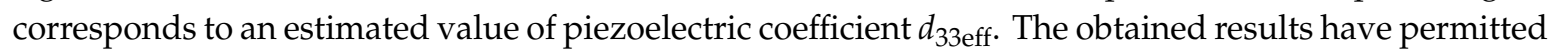


to estimate the $d_{33 \text { eff }}$ values of composite fibers with varying concentration of graphene oxide (Figure 7 , Table 1).
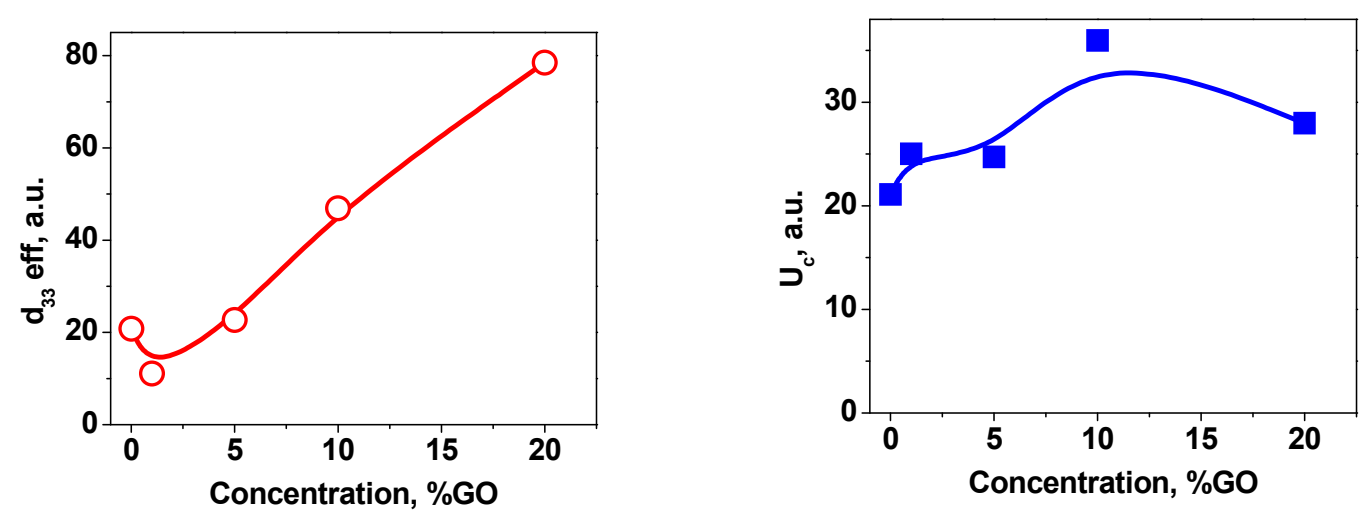

Figure 7. Concentration dependences of switching parameters of $\mathrm{P}(\mathrm{VDF}-\mathrm{TrFE})$-graphene oxide fibers with different concentration of GO.

Table 1. Concentration dependence of piezoelectric coefficient of P(VDF-TrFE)-graphene oxide fibers with different concentration of GO.

\begin{tabular}{ccc}
\hline GO, $\%$ & $\boldsymbol{U}_{\mathrm{c}}, \mathbf{V}$ & $\boldsymbol{d}_{\mathbf{3 3}}$, a.u. \\
\hline 0 & 21.1 & 20.8 \\
1 & 25 & 11.1 \\
5 & 24.7 & 22.7 \\
10 & 35.95 & 46.9 \\
20 & 27.95 & 78.5 \\
\hline
\end{tabular}

One of the most important observations is the fact of stability of the poled state of fibers (Figure 8). It was observed that no ferroelectric domains in the initial time. Voltages were applied at the central point of fiber. The fibers was subjected to poling with $-75 \mathrm{~V} D C$ (direct current) bias. The PFM images after poling composite fibers with varying concentration of GO are shown in Figure 8. The regions of uniform polarization within the fiber are rough and irregular. In addition, no direct correlation between the piezoelectric contrast and topographic shape is evident, which confirms that the observed effect is the intrinsic piezoelectric property of the fibers and it has not originated from electrostatic effect and/or cross-talk with topography. The influence of the concentration of the GO on the length of domain switching is shown in Figure 9. 

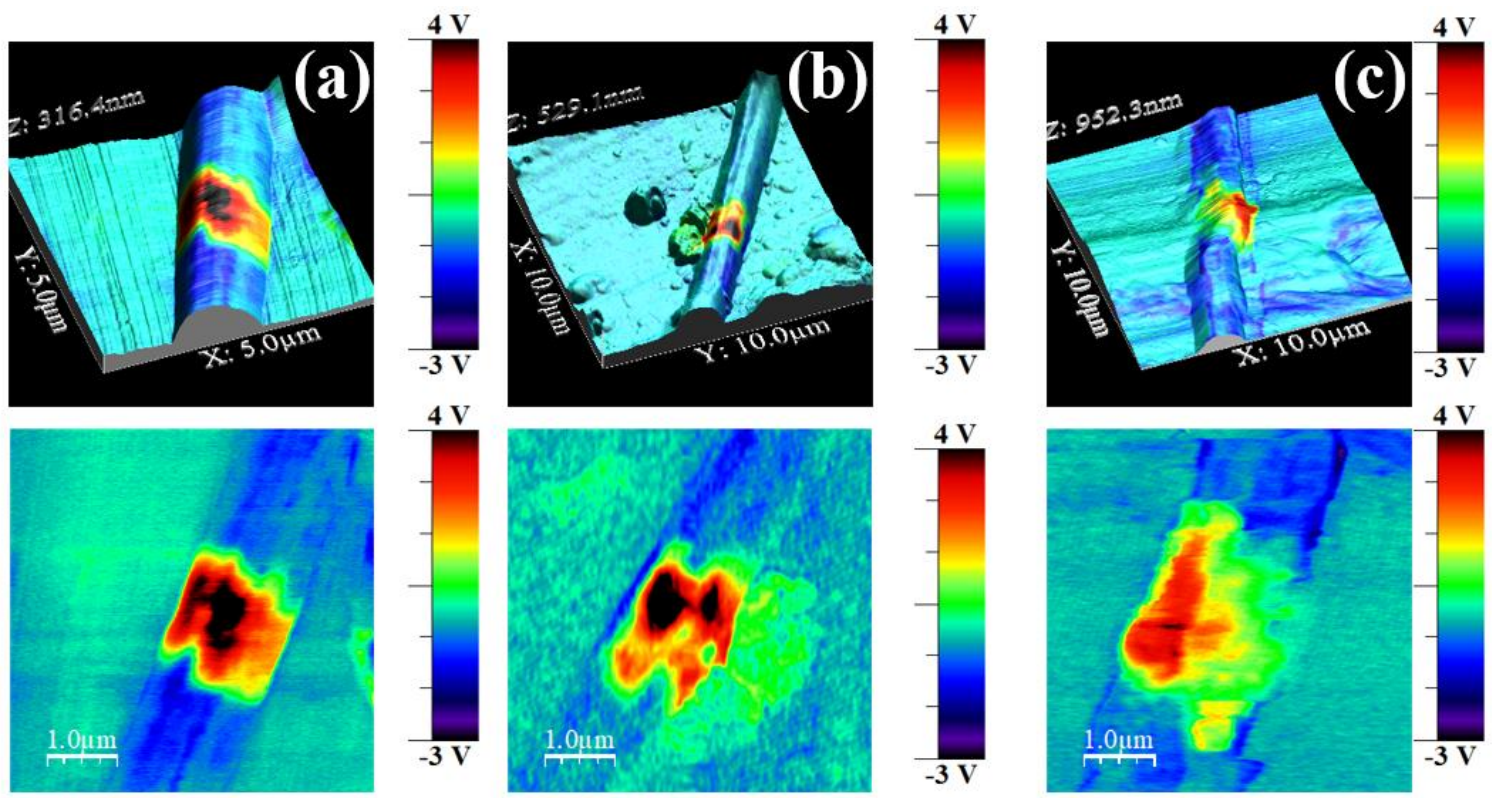

Figure 8. PFM images after poling $(\mathrm{U}=-75 \mathrm{~V}, \mathrm{t}=50 \mathrm{~s})$.

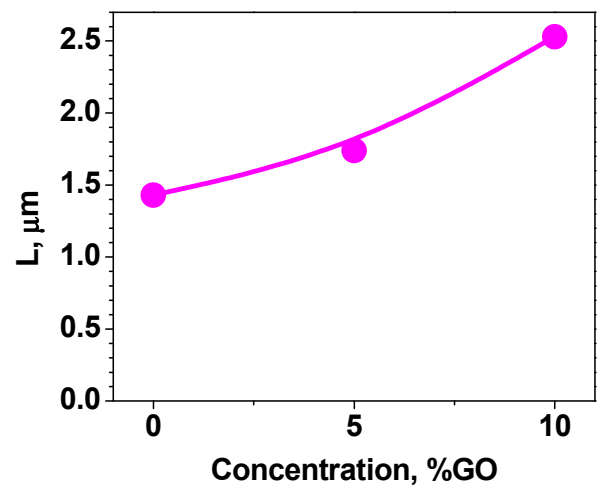

Figure 9. Concentration dependence length of domain after poling $(U=-75 \mathrm{~V}, \mathrm{t}=50 \mathrm{~s})$.

For understanding the mechanism of piezoresponse evolution in these composites, using our previous models of PVDF chain (Figure 10a) and their behavior in electric fields (Figure 10b) we have computed piezoelectric coefficients [26]. It is the case of the previous flat models, which corresponds to PVDF films samples. The curved PVDF chain model (Figure 10c) is proposed and considered for the case of the fibers samples. The symmetrized models of graphene oxide $(\mathrm{GO})$ based on graphene $(\mathrm{G})$ layer consisting of 54 carbon atoms (G54), 96 carbon atoms (G96) and arranged with hydrogens (G54H, $\mathrm{G} 96 \mathrm{H}$ ) are considered similarly as in our previous work [26]: with oxygen and $\mathrm{OH}$ groups (Figure 11a) (formula $\mathrm{Gr}_{96} \mathrm{HN}_{2} \mathrm{O}_{2} \mathrm{H}_{2}$ and further abbreviation $\mathrm{GO}_{2}$ ) and with $\mathrm{COOH}$ groups (Figure 11b) (formula $\mathrm{Gr}_{96} \mathrm{~N}_{2} \mathrm{O}_{2} \mathrm{H}_{2} \mathrm{COOH}_{2}$, abbreviation GO1). 


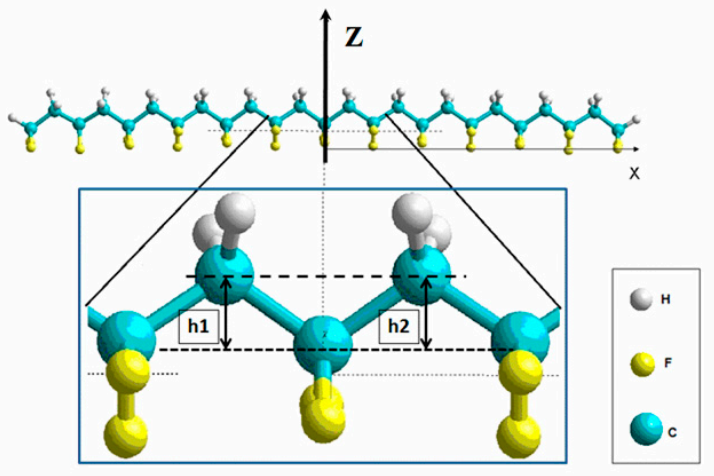

(a)

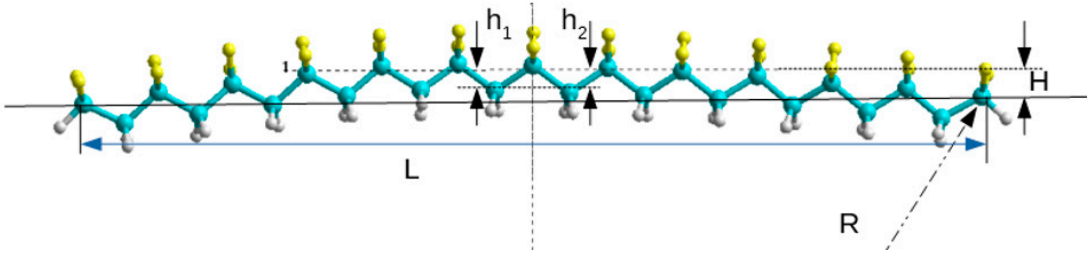

(c)

Figure 10. Model of PVDF chain: (a) PVDF and (b) Its central part, (c) Model of curved PVDF chain ( $R$-radius of curvature, $L$-chord of curvature, $H$-height of curved chain on the length of chord $L$; $h_{1}$ and $h_{2}$ are similar as on above Figure $10 \mathrm{~b}$ ).

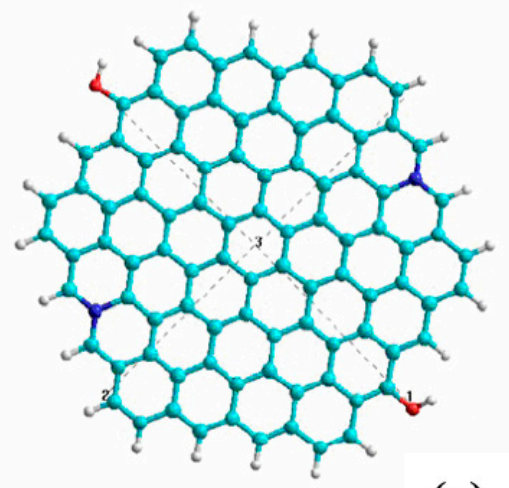

(a)

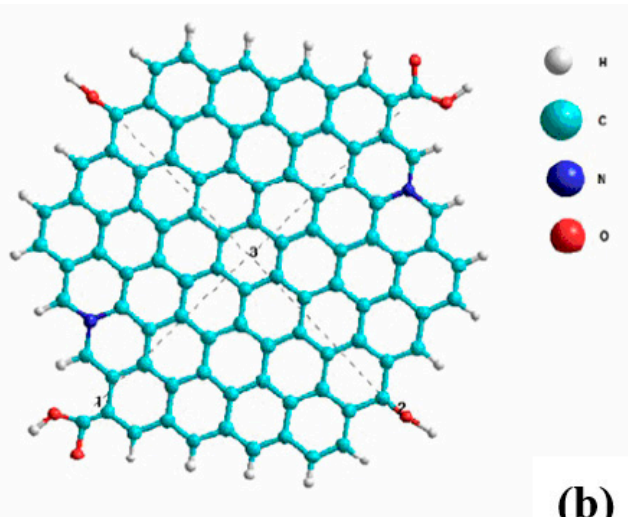

(b)

Figure 11. Graphene oxide layers models: (a) with $\mathrm{OH}$ group (GO2 model); (b) with $\mathrm{COOH}$ group (GO1 model).

Several simplest models for the PVDF/graphene oxide complex were proposed and tested (Figure 12) similarly to our previous work [25]. The piezoelectric coefficients for these models were calculated using similar calculation algorithms, as was done in works $[25,26]$. Thus, the following three models were constructed and numerically investigated, viz. \#1-with side $\mathrm{H}$ (hydrogen atoms) included in the PVDF chain and located towards the graphene oxide layer, \#2-with side F (fluorine atoms) included in the PVDF chain and located towards the graphene oxide layer (both of these first options show approximately the same values of the calculated piezoelectric coefficients) and \#3-graphene oxide layers on both sides surrounds the PVDF chain (sandwich-type model), as shown in Figure 12a,b. 


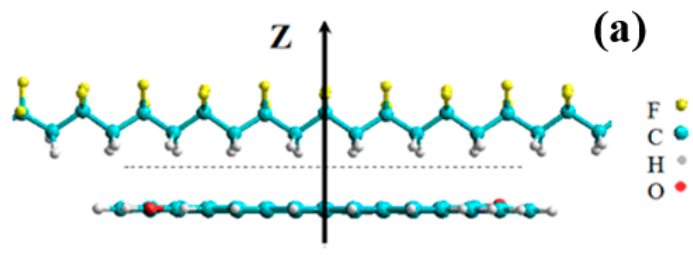

(c)
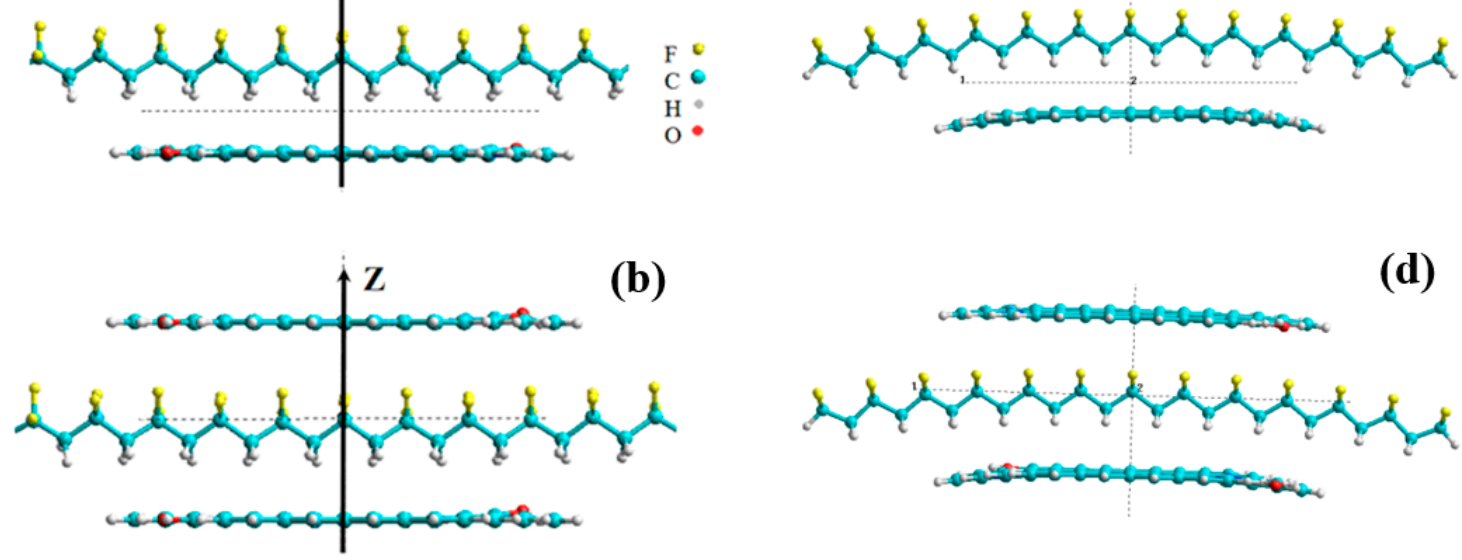

(d)

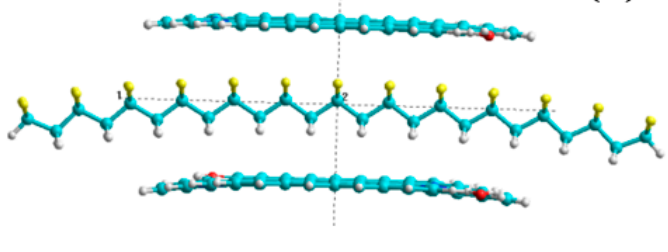

Figure 12. Models of PVDF/graphene oxide structures: (a) with H-side, flat PVDF/graphene oxide model (GO with G96H model with OH groups); (b) flat sandwich model for graphene oxide/PVDF/graphene oxide (the same case of G96H); PVDF curved chain models with determination the geometry of used radius $\mathrm{R}$ and chord L configuration (see on Figure 10c); (c) with one H-side case, PVDF/graphene curved model-(PVDF + G96H or PVDF/G96H) (graphene from 96 carbon atoms arranged by hydrogen atoms); (d) a curved sandwich model with two layers of $\mathrm{GO}$ from $\mathrm{G} 96 \mathrm{H}$ and $\mathrm{OH}$ group for the graphene oxide/PVDF/graphene oxide-(PVDF+2 $\mathrm{Gr} 96 \mathrm{HN}_{2} \mathrm{O}_{2} \mathrm{H}_{2}$ or $\left.\mathrm{GO} 2 / \mathrm{PVDF} / \mathrm{GO} 2\right)$.

For a deeper understanding, the case of the composite fibers formation, we also considered two versions of the graphene layers in relation with PVDF chain: similar as the flat models-the same configuration of the curved models (Figure 12c,d) models with one side and two sides sandwich models. It should be noted that the cluster geometry of the mentioned models is similar to the situation with graphene oxide layers the while the latter case is much more difficult in terms of calculations. The results obtained after modeling and calculations are presented below in Table 2.

Table 2. The piezoelectric coefficients $d_{33}$ calculated for different types of structures (flat and curved) of Graphene Oxide with and PVDF (in comparison with the data from other works [24,25], in an electric field $\mathrm{Ez} \sim 500 \mathrm{GV} / \mathrm{m}$, and with experimental data obtained in the present study; the presented values have absolute value (without natural negative sign).

\begin{tabular}{|c|c|c|c|c|c|}
\hline$\#$ & Model Contents & $\begin{array}{c}\text { Flat Models* } \\
d_{33}, \mathrm{pm} / \mathrm{V}\end{array}$ & $\begin{array}{c}\text { Films } \\
d_{33}, \text { a.u., } \mathrm{V} \\
\text { (Experimental) }\end{array}$ & $\begin{array}{c}\text { Curved } \\
\text { Models } \\
d_{33, \mathrm{pm} / \mathrm{V}}\end{array}$ & $\begin{array}{c}\text { Fibers } \\
d_{33}, \text { a.u. } \\
\text { (Experimental) }\end{array}$ \\
\hline 0 & PVDF pure (chain) & $38.5 * *$ & 7.5 & 22.3 & 20.8 \\
\hline 1 & PVDF/G54 1 side & 12.2 & - & - & - \\
\hline 2 & PVDF/G54H 1 side & 9.8 & - & 16.7 & 11.1 \\
\hline 3 & $\begin{array}{l}\text { G54H/PVDF/G54H } \\
2 \text { sides (sandwich) }\end{array}$ & 19.0 & - & 34.8 & 22.7 \\
\hline 4 & $\begin{array}{l}\text { G96H/PVDF/G96H } \\
2 \text { sides (sandwich) }\end{array}$ & - & - & 51.9 & 46.9 \\
\hline 5 & PVDF/GO1 1 side & 14.6 & 5.9 & & \\
\hline 6 & PVDF/GO2 1 side & 13.5 & - & & \\
\hline 7 & $\begin{array}{c}\text { GO1/PVDF/GO1 } \\
2 \text { sides (sandwich) }\end{array}$ & 18.7 & 6.7 & & \\
\hline 8 & $\begin{array}{c}\text { GO2/PVDF/GO2 } \\
2 \text { sides (sandwich) }\end{array}$ & 29.8 & & & \\
\hline
\end{tabular}

* [25], ** [24].

An optimization strategy which determines the changes in the atomic configuration of studied clusters $[24,25]$ assumes several models, the first one considers an absence of electric field which allows to find the initial optimal atomic positions of modeled composite structure and to determine the initial 
optimal parameters of PVDF chain heights in its central part $\left(h_{1}\right.$, and $\left.h_{2}\right)$. The second model considers an external electric field with $\mathrm{Z}$ orientation (along the main polarization vector of PVDF), this model allows to find out the optimal geometry for new atomic configuration under action of electric field. The third model assumes the changes of the main parameters $\left(h_{1}\right.$ and $h_{2}$, Figure 10) from initial optimal parameters, which allows to determine the deformation $\Delta h_{1}$ and $\Delta h_{2}$, and to calculate the corresponding values of voltage $U$. Finally, the piezoelectric coefficient $d_{33}$ was computed, using dielectric permittivity value of $\varepsilon=10$. Comparison with the data known for $d_{33}$ attributed to initial PVDF samples testifies that under the influence of graphene oxide layer the piezoelectric coefficient $d_{33}$ of new composite structure is decreased. It has nearly three times lower value: $d_{33}=-14.6 \mathrm{pm} / \mathrm{V}$ (or pC/N) for the simple flat models (with one side H or F of PVDF chain-Figure 12a), which interacting with one GO layer (with OH groups), as compared with the average value of the pure PVDF $d_{33}=-38.5 \mathrm{pm} / \mathrm{V}(\mathrm{pC} / \mathrm{N})$. It is important to note, that the sign of $d_{33}$ coefficient is negative in all cases as it was established in [26,27] for the initial pure PVDF, it is caused by specific mechanism of PVDF chain deformation under an applied electric field. In the case of flat with double graphene oxide layers (sandwich model structure) the piezoelectric coefficient $d_{33}$ is increased to the value of $d_{33}=-29.8 \mathrm{pm} / \mathrm{V}(\mathrm{pC} / \mathrm{N})$ (Figure 12b, and see in Table 2, data for flat models).

The experimental results qualitatively correlate with those obtained in the calculations (see in Figures 7 and 13 and Table 2). We assume that the experimental data obtained for the composite with small amount of graphene oxide correlate to the model constructed for the PVDF chain interacted with graphene oxide from one side only. The results obtained for this case show a reduction in the piezo-response as well as in the piezoelectric coefficient. From the other side, piezoelectric properties of the composites with larger GO content correspond to the model assuming the sandwich-like clusters in the composite. The experimental data testify a decrease in the piezoelectric signal in these composites which is caused by statistical dispersion and disorientation of graphene oxide layers and PVDF chains (or layers). Uncontrolled thickness of the individual layers of graphene oxide and PVDF component can also affect piezoelectric properties of the composites. Statistical disorder obtained in these cases could not yield an exact match with the simulation performed. However, even at low concentrations, the effect of molecular ordering is observed, and for $20 \%$ GO composite the probability of the formation of sandwich structures (as proposed in our modeling) is quite significant. Furthermore, in the case of controlled hetero-structures we can consider a much greater effect and results will be closer to experimental.

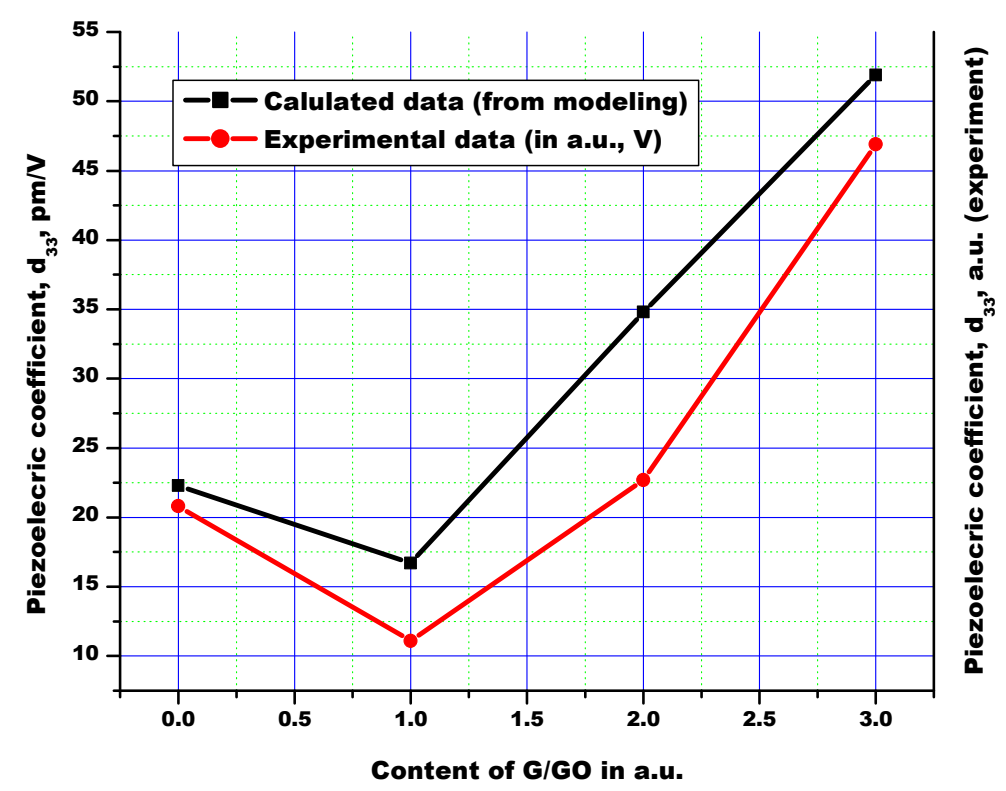

Figure 13. Trend of changes for computed $d_{33}$ using curved models of PVDF + Graphene as compared to the experimental data. 
Abbreviations used in Table 2 are the following: GO1 is graphene oxide which contain $\mathrm{COOH}$ groups $\left(\mathrm{Gr}_{6} \mathrm{~N}_{2} \mathrm{O}_{2} \mathrm{H}_{2} \mathrm{COOH}_{2}\right.$, which was abbreviated as $\mathrm{Gr} 96 \mathrm{NO}$ in [27]), $\mathrm{GO} 2$ is graphene oxide with $\mathrm{OH}$ group written as formula $\mathrm{Gr} 96 \mathrm{HN}_{2} \mathrm{O}_{2} \mathrm{H}_{2}$ above.

We try to consider and discussed here also the case of model with curved PVDF chain and Graphene layers $[25,26]$. This situation could be corresponding to the fiber's surface-it is a curved surface with some radius $\mathrm{R}$. In the experimental case it is approximately $\mathrm{R} \sim 50 \ldots 100 \mathrm{~nm}$. But in our modeling, we use approximately $\mathrm{R} \sim 10 \mathrm{~nm}$ (and more) for most bright and expressive influence of such curvatures, which imitates the curved surface. This radius could be simply determined from usual geometry using formula (see about of some geometrical chord (with high $H$ and length $L$ ) determinations on Figure 10c:

$$
\mathrm{R}=\left(\mathrm{H}^{2}+\mathrm{L}^{2} / 4\right) /(2 \mathrm{H})
$$

It is known from the literature that for the case of curved (or flexible) polymers such as PVDF and related materials, the piezoelectric coefficients are changed in this case [27-30]. But all computed data used here for our calculation of $d_{33}$ and presented on Figure $11 \mathrm{c}, \mathrm{d}$ the same as was shown on Figure 9 (and used in our previous papers [29,30]). On Figure 12c,d are shown the series of such curved models in this case similar as in our previous models (see on Figures 9-11) and following results of calculated piezoelectric coefficients are presented in Table 2 (last two columns for curved models) and on Figure 13.

We must note that in this situation is very hard convergence of computed data for these curved models during optimization process (searching of the minimum of total energy surface for these complex molecular models using conjugate gradient methods) due their more complex structures. This work will be continued to get better accuracy of the calculations. But now we have only the case of pure Graphene layers (not the Graphene Oxide models-as it is also more complicated for optimization of curved models) and yet have some wide scatter of the data for this case. It should be noted that different types of oxidation $(\mathrm{O}, \mathrm{OH}, \mathrm{COOH}$ etc.) may notably change the functional properties of the composites and appropriate calculations becomes to be quite difficult for conventional computer modelling while these models will be considered in our further works. However, despite on used different units for values of the piezoelectric coefficient, current results clearly show the same trend of changes both for the computed and measured data (see on Figures 6 and 13 and Tables 1 and 2).

\section{Conclusions}

Fibers with different concentrations of GO were successfully prepared and the influence on the electric potential and piezoelectric coefficient of $\mathrm{P}(\mathrm{VDF}-\mathrm{TrFE})$ copolymer are investigated. An increase in local piezoelectric response with increasing graphene oxide concentration in the composite fibers was found. This corresponds to the theoretical modeling and results obtained on the same composite thin films both as flat and as curved ones. In addition, the experimental results suggested a dependence of electric potential on the density of the PVDF-GO fibers. The obtained results are in accordance with the data declared in some recent works of other authors [31,32].

Author Contributions: Data curation, D.Z., M.B., P.M.V. and B.S.; Formal analysis, V.B.; Investigation, D.K., M.S. and I.B.; Supervision, P.A.A.P.M.; Writing-original draft, V.B.

Acknowledgments: M.S. acknowledges Russian academic excellence project "5-100" for Sechenov University.

Conflicts of Interest: The authors declare no conflict of interest.

\section{References}

1. Fuh, Y.K.; Wang, B.S. Near field sequentially electrospun three-dimensional piezoelectric fibers arrays for self-powered sensors of human gesture recognition. Nano Energy 2016, 30, 677-683. [CrossRef]

2. Wang, L.-Y.; Yong, W.F.; Yu, L.E.; Chung, T.-S.; Chung, N.T.-S. Design of high efficiency PVDF-PEG hollow fibers for air filtration of ultrafine particles. J. Membr. Sci. 2017, 535, 342-349. [CrossRef] 
3. Wu, Z.; Cui, Z.; Li, T.; Qin, S.; He, B.; Han, N.; Li, J. Fabrication of PVDF-based blend membrane with a thin hydrophilic deposition layer and a network structure supporting layer via the thermally induced phase separation followed by non-solvent induced phase separation process. Appl. Surf. Sci. 2017, 419, 429-438. [CrossRef]

4. Huang, Z.-X.; Liu, X.; Wong, S.-C.; Qu, J.-P. Electrospinning polyvinylidene fluoride/expanded graphite composite membranes as high efficiency and reusable water harvester. Mater. Lett. 2017, 202, 78-81. [CrossRef]

5. Wang, X.; Wang, Z.; Wang, Z.; Cao, Y.; Meng, J. Tethering of hyperbranched polyols using PEI as a building block to synthesize antifouling PVDF membranes. Appl. Surf. Sci. 2017, 419, 546-556. [CrossRef]

6. Abdallah, H. A Review on Catalytic Membranes Production and Applications. Bull. Chem. React. Eng. Catal. 2017, 12, 136-156. [CrossRef]

7. Gong, C.; Liu, H.; Zhang, B.; Wang, G.; Cheng, F.; Zheng, G.; Wen, S.; Xue, Z.; Xie, X. High level of solid superacid coated poly (vinylidene fluoride) electrospun nanofiber composite polymer electrolyte membranes. J. Membr. Sci. 2017, 535, 113-121. [CrossRef]

8. Arbab, A.A.; Peerzada, M.H.; Sahito, I.A.; Jeong, S.H. A complete carbon counter electrode for high performance quasi solid state dye sensitized solar cell. J. Power Sources 2017, 343, 412-423. [CrossRef]

9. Terasaw, N.; Asak, K. Superior performance of PEDOT: Poly (4-styrenesulfonate)/vapor-grown carbon fibre/ionicliquid actuators exhibiting synergistic effects. Sens. Actuators B 2017, 248, 273-279. [CrossRef]

10. Thakur, N.; Ranganath, A.S.; Agarwal, K.; Baji, A. Electrospun Bead-On-String Hierarchical Fibers for Fog Harvesting Application. Macromol. Mater. Eng. 2017, 302, 1700124. [CrossRef]

11. Ren, X.; Fan, H.; Wang, C.; Ma, J.; Lei, S.; Zhao, Y.; Li, H.; Zhao, N. Magnetic force driven noncontact electromagnetic-triboelectric hybrid nanogenerator for scavenging biomechanical energy. Nano Energy 2017, 35, 233-241. [CrossRef]

12. Liu, X.; Kuang, X.; Xu, S.; Wang, X. High-sensitivity piezoresponse force microscopy studies of single polyvinylidene fluoride nanofibers. Mater. Lett. 2017, 191, 189-192. [CrossRef]

13. Lu, X.; Qu, H.; Skorobogatiy, M. Piezoelectric Micro- and Nanostructured Fibers Fabricated from Thermoplastic Nanocomposites Using a Fiber Drawing Technique: Comparative Study and Potential Applications. ACS Nano 2017, 11, 2103-2114. [CrossRef] [PubMed]

14. Matsouka, D.; Vassiliadis, S.; Bayramol, D.V.; Soin, N.; Siores, E. Investigation of the durability and stability of piezoelectric textile fibres. J. Intell. Mater. Syst. Struct. 2016, 28, 663-670. [CrossRef]

15. Bodkhe, S.; Turcot, G.; Gosselin, F.P.; Therriault, D. One-Step Solvent Evaporation-Assisted 3D Printing of Piezoelectric PVDF Nanocomposite Structures. ACS Appl. Mater. Interfaces 2017, 9, 20833-20842. [CrossRef] [PubMed]

16. Abolhasani, M.M.; Shirvanimoghaddam, K.; Naebe, M. PVDF/graphene composite nanofibers with enhanced piezoelectric performance for development of robust nanogenerators. Compos. Sci. Technol. 2017, 138, 49-56. [CrossRef]

17. Huang, T.; Lu, M.; Yu, H.; Zhang, Q.; Wang, H.; Zhu, M. Enhanced Power Output of a Triboelectric Nanogenerator Composed of Electrospun Nanofiber Mats Doped with Graphene Oxide. Sci. Rep. 2015, 5, 13942. [CrossRef] [PubMed]

18. Mokhtari, F.; Latifi, M.; Shamshirsaz, M.; Khelghatdoost, M.; Rahmani, S. Modeling of electrospun PVDF/LiCl nanogenerator by the energy approach method: determining piezoelectric constant. J. Text. Inst. 2017, 108, 1917-1925. [CrossRef]

19. Gonçalves, G.; Marques, P.A.A.P.; Granadeiro, C.M.; Nogueira, H.I.S.; Singh, M.K.; Grácio, J.; Nogueira, H.S. Surface Modification of Graphene Nanosheets with Gold Nanoparticles: The Role of Oxygen Moieties at Graphene Surface on Gold Nucleation and Growth. Chem. Mater. 2009, 21, 4796-4802. [CrossRef]

20. Roelofs, A.; Schneller, T.; Szot, K.; Waser, R. Piezoresponse force microscopy of lead titanate nanograins possibly reaching the limit of ferroelectricity. Appl. Phys. Lett. 2002, 81, 5231-5233. [CrossRef]

21. HyperChem (TM) Professional 7.51, Tools for Molecular Modeling; HyperChem 8.0, Professional Edition; Hypercube. Inc.: Gainesville, FL, USA, 2010.

22. Bader, F.W.; Nguyen-Dang, T.T. Quantum theory of atoms in molecules-Dalton revisited. Adv. Quantum Chem. 1981, 14, 63-124.

23. Huang, L.; Lu, C.; Wang, F.; Wang, L. Preparation of PVDF/graphene ferroelectric composite films by in situ reduction with hydrobromic acids and their properties. RSC Adv. 2014, 4, 45220-45229. [CrossRef] 
24. Melitz, W.; Shen, J.; Kummel, A.C.; Lee, S. Kelvin probe force microscopy and its application. Surf. Sci. Rep. 2011, 66, 1-27. [CrossRef]

25. Bystrov, V.S.; Paramonova, E.V.; Bdikin, I.K.; Bystrova, A.V.; Pullar, R.C.; Kholkin, A.L. Molecular modeling of the piezoelectric effect in the ferroelectric polymer poly(vinylidene fluoride) (PVDF). J. Mol. Model. 2013, 19, 3591-3602. [CrossRef] [PubMed]

26. Bystrov, V.S.; Bdikin, I.K.; Silibin, M.; Karpinsky, D.; Kopyl, S.; Paramonova, E.V.; Goncalves, G. Molecular modeling of the piezoelectric properties in the Graphene/Graphene oxide and polyvinylidene fluoride (PVDF) polymer ferroelectric composites. J. Mol. Mod. 2017, 23, 128. [CrossRef] [PubMed]

27. You, S.; Shi, H.; Wu, J.; Shan, L.; Guo, S.; Dong, S. A flexible, wave-shaped P(VDF-TrFE)/metglas piezoelectric composite for wearable applications. J. Appl. Phys. 2016, 120, 234103. [CrossRef]

28. Li, G.; Furman, E.; Haertling, G.H. Stress-Enhanced Displacements in PLZT Rainbow Actuators. J. Am. Ceram. Soc. 1997, 80, 1382. [CrossRef]

29. Schwartz, R.W.; Moon, Y. Domain configuration and switching contributions to the enhanced performance of rainbow actuators. In Smart Structures and Materials 2001: Active Materials: Behavior and Mechanics; International Society for Optics and Photonics: Bellingham, WA, USA, 2001; Volume 4333, pp. 408-418.

30. Kang, L.-H.; Han, J.-H. Prediction of actuation displacement and the force of a pre-stressed piezoelectric unimorph (PUMPS) considering nonlinear piezoelectric coefficient and elastic modulus. Smart Mater. Struct. 2010, 19, 94006. [CrossRef]

31. Viskadouros, G.M.; Stylianakis, M.M.; Kymakis, E.; Stratakis, E. Enhanced Field Emission from Reduced Graphene Oxide Polymer Composites. ACS Appl. Mater. Interfaces 2013, 6, 388-393. [CrossRef]

32. Stylianakis, M.M.; Viskadouros, G.; Polyzoidis, C.; Veisakis, G.; Kenanakis, G.; Kornilios, N.; Petridis, K.; Kymakis, E. Updating the Role of Reduced Graphene Oxide Ink on Field Emission Devices in Synergy with Charge Transfer Materials. Nanomaterials 2019, 9, 137. [CrossRef]

(C) 2019 by the authors. Licensee MDPI, Basel, Switzerland. This article is an open access article distributed under the terms and conditions of the Creative Commons Attribution (CC BY) license (http://creativecommons.org/licenses/by/4.0/). 